\title{
A thermal instability for positive brane cosmological constant in the Randall-Sundrum cosmologies
}

\author{
Stephon Alexander ${ }^{2,3}$, Yi Ling ${ }^{1,2 \dagger}$, Lee Smolin*1,2* \\ ${ }^{1}$ Center for Gravitational Physics and Geometry, Department of Physics, \\ The Pennsylvania State University, University Park, PA, USA 16802. \\ ${ }^{2}$ The Blackett Laboratory, Imperial College of Science, Technology and Medicine, London SW7 2BZ, UK \\ ${ }^{3}$ Institute for Strings, Cosmology and Astroparticle Physics, Columbia University, New York, NY 10027, USA
}

November 5, 2018

\begin{abstract}
We describe a novel dynamical mechanism to radiate away a positive four dimensional cosmological constant, in the Randall-Sundrum cosmological scenario. We show that there are modes of the bulk gravitational field for which the brane is effectively a mirror. This will generally give rise to an emission of thermal radiation from the brane into the bulk. The temperature turns out to be nonvanishing only if the effective four dimensional cosmological constant is positive. In any theory where the four dimensional vacuum energy is a function of physical degrees of freedom, there is then a mechanism that radiates away any positive four dimensional cosmological constant.
\end{abstract}

\section{Introduction}

In this letter we describe a new dynamical mechanism, which, in the context of the RandallSundrum cosmological scenario [1, 2, 3, 团, renders unstable solutions described by the embedding of a four dimensional de Sitter universe in the five dimensional AdS spacetime. This mechanism is closely related to the Unruh[0] effect and, more directly, to the effect whereby accelerated mirrors radiate[ [6, 团]. When the four dimensional vacuum energy (or cosmological

*email: s.alexander@ic.ac.uk, ${ }^{\dagger}$ ling@phys.psu.edu, * smolin@phys.psu.edu 
constant) is a function of dynamical degrees of freedom, such as when the vacuum energy depends on the value of a scalar field, our result implies that positive vacuum energy density may be radiated into the bulk, resulting in a decrease of the effective four dimensional cosmological constant円.

More particularly, we describe here three results of an investigation into the possible role of Unruh and moving mirror acceleration in the context of the Randall-Sundrum cosmologies.

- There are modes of the linearized bulk gravitational field which, rather than being bound to the brane, see the brane as a perfectly reflecting mirror.

- Given plausible physical assumptions, this implies that the brane does radiate into the $A d S$ bulk spacetime. However, when the effective four dimensional cosmological constant $\lambda_{4}$ vanishes, or is negative, the temperature is zero. Thus, the original RS scenario in which $\lambda_{4 e}=0$ is in fact stable and does not Unruh radiate into the bulk.

- When $\lambda_{4 e}$ is strictly positive, the temperature is non-vanishing. The temperature is proportional to the square root of effective $4 \mathrm{~d}$ cosmological constant, $\sqrt{\lambda_{4 e}}$.

We note that so long as the approximations we employ are valid, these results mean that any positive value of the cosmological constant can be radiated away, with a temperature governed by $\sqrt{\lambda_{4 e}}$ ?.

In the next section we display the existence of linearized modes of the bulk gravitational field which see the brane as a mirror. In section 3 we apply to this known results on Unruh radiation in $A d S$ spacetime and moving mirrors to deduce the conclusions just mentioned. In the concluding section we discuss the range of parameters in which our approximations may be considered valid.

\footnotetext{
${ }^{1}$ Earlier proposals for dynamical decay of the cosmological constant in de Sitter spacetime were described in $[8]$.

${ }^{2}$ Chamblin et al [9], previously discussed moving mirror radiation from branes and its interaction with Hawking radiation from black holes in the bulk. A related idea, studied previously by Deffayet et al 10 and Levin[11], is that de Sitter radiation in the brane might leak into the bulk, resulting in decay of the cosmological constant.
} 


\section{The Brane as a Mirror}

Randall and Sundrum and others have studied modes of the linearized bulk Einstein equations which are, in the sense described in [3], bound to the brane. In this section we demonstrate the existence of other linearized bulk modes, for which the brane functions as a mirror. We begin with the five dimensional action for the RS scenario:

$$
S=\frac{1}{G_{5}} \int d^{5} x \sqrt{-g_{5}}\left(\mathcal{R}^{(5)}-\Lambda\right)-\int \mathrm{d}^{4} \mathrm{x} \sqrt{-\mathrm{g}_{4}}\left(\tau+\mathcal{L}_{\text {matter }}\right),
$$

where $\mathcal{R}^{(5)}$ is the five dimensional Ricci scalar, $g_{5}$ and $g_{4}$ are the five and four dimensional metrics respectively, $G_{5}=l_{P l}^{2} R$ is the five dimensional Newton's constant and $\tau$ is the brane tension. The bulk space is a piece of anti-de Sitter space described by

$$
d s^{2}=d y^{2}+e^{-|y| / R} \eta_{\mu \nu} d x^{\mu} d x^{\nu},
$$

and the brane resides at $y=0$. We are interested in the boundary conditions for gravitational modes at the brane. Hence, we begin by linearizing the Einstein equations.

We begin by noting that it is possible to consider two classes of bulk perturbations. The first are those identified by Randall-Sundrum as those bound to the brane [3].

$$
d s^{2}=d y^{2}+e^{-|y| / R}\left[\eta_{\mu \nu}+\gamma_{\mu \nu}\right] d x^{\mu} d x^{\nu},
$$

where $\gamma_{\mu \nu}$ is assumed to be $C^{\infty}$ at the brane. This means that the derivatives of the linearized

modes actually have singular behavior near the brane, coming from terms in $\partial_{y}|y|$ and $\partial_{y}^{2}|y|$ at $y=0$. The behavior of these modes is by now well studied to linearized order. Let us call these type I modes.

These are not the only linearized modes of the metric in the spacetime. Consider perturbations of the form,

$$
d s^{2}=d y^{2}+\left[e^{-|y| / R} \eta_{\mu \nu}+h_{\mu \nu}\right] d x^{\mu} d x^{\nu},
$$

where now $h_{\mu \nu}(y, x)$ is considered to be non-singular at the brane. Let us call these type II modes. To study them it is more convenient to switch to coordinates $z=R e^{y / R}$, for $y \geq 0$ (which implies that $z \geq R$ ), so that the $A d S$ metric is of the form

$$
d s^{2}=\frac{R^{2}}{z^{2}}\left(d z^{2}+d x_{4}^{2}\right)
$$


and the linearized metric is of the form,

$$
d s^{2}=\frac{R^{2}}{z^{2}}\left(d z^{2}+d x_{4}^{2}\right)+h_{\mu \nu}(z, x) d x^{\mu} d x^{\nu} .
$$

Again $h_{\mu \nu}$ is assumed to be non-singular'f at the position of the brane, which is now $z=R$.

We choose a flat brane located at $z=z_{0}=R$ with $R^{2}=\Lambda$ and then linearize the field equations arising from the action (1). It is sufficient for our purposes to go directly to traceless, transverse gauge, where we find,

$$
\frac{1}{\sqrt{-g_{5}}} \partial_{\rho} g_{5}^{\rho \sigma} \partial_{\sigma} \sqrt{-g_{5}} h_{\mu \nu}=\tau \delta\left(z, z_{0}\right) h_{\mu \nu}+T_{\mu \nu}^{\text {matter }} \delta\left(z, z_{0}\right)
$$

Since the brane tension $\tau$ is non-vanishing, all solutions to this equation in the absence of matter on the brane must satisfy

$$
h_{\mu \nu}\left(z_{0}\right)=0 .
$$

This is simply the Dirichlet boundary condition. Thus, we find that type II modes which satisfy the linearized Einstein's equations exist, but they are not free on the brane, instead, in the absence of matter, the brane acts as a mirror for these modest

This result is not special to the flat embedding, it applies to all fluctuations around brane embeddings which extremize the action (1) of the form,

$$
d s^{2}=d y^{2}+\left[g_{\mu \nu}^{0}+h_{\mu \nu}\right] d x^{\mu} d x^{\nu}
$$

where $g_{\mu \nu}^{0}$ is the metric for an embedding of a brane.

Type II modes were considered also by Randall and Sundrum[3], who argued that they interact very weakly with matter on the brane. While this is true, we will show below that

\footnotetext{
${ }^{3}$ Note that while the background RS solution is invariant under a parity transformation in which $y \rightarrow-y$, the same is not required to be true of the linearization of solutions close to the RS solution. The point is that while a particular solution to Einstein's equations may have an isometry, no isometry can be imposed on the whole space of solutions to the Einstein's equations. (In fact, only a set of measure zero of metrics in the space of solutions have any isometries). Thus, if we consider linearizing the Einstein's equations around a given solution with an isometry, the isometry cannot be imposed also on the linearized modes. The reason is that to be physically meaningful the linearized modes must span the space of linearizations of exact solutions close to the original one. Were we to impose a symmetry of the background on the linearized modes we might miss some modes which are linearizations of full solutions. Thus we must consider all type II modes which satisfy the boundary conditions, without regard to symmetry.

${ }^{4}$ Note that for Type I modes the dangerous $\tau \delta\left(z, z_{0}\right)$ term is canceled by a delta function coming from the term $\partial_{y}^{2}|y| / R$ which comes from the five dimensional wave operator applied to the perturbation $e^{-|y| / R} h_{\mu \nu}$.
} 
the fact that they see the brane as a mirror has consequences due to the quantum effect that accelerated mirrors in some circumstances radiate.

Before going on to understand what the consequences of the type II modes are for the RS cosmological scenarios we should comment on the existence of two kinds of modes of the linearized metric, Type I, bound to the brane and Type II, which are reflected by the brane. How can we tell if either, or both are true modes of the gravitational field? The point is that not any solution to the linearized Einstein's equation represents an actual linearization of a full solution to the full Einstein's equations. What is required is more [12]: $h_{\mu \nu}$ is a genuine linearization of a full solution to Einstein's equations if there is a one parameter family of exact solutions $g_{\mu \nu}(\lambda), \phi(\lambda)$, where $\phi$ represents all the matter degrees of freedom, such that $d g_{\mu \nu}(\lambda) /\left.d \lambda\right|_{\lambda=0}=h_{\mu \nu}$. One simple check is to carry out an expansion of the Einstein equations to higher order, to show that a solution can be generated order by order. In the case of Type I modes these equations may be singular as a result of the $\partial_{y}|y| \approx \theta(y)$ and $\partial_{y}^{2}|y| \approx \delta(y)$ behavior of the derivatives of the modes at the location of the brane at $y=0$. This is a question that deserves investigation.

\section{The Brane Mirror and Thermal Radiation}

The problem of moving mirror boundary conditions in quantum field theory has been studied in some detail since the 1970's[6, 6]. The conclusion in Minkowski spacetime is that a moving mirror with a constant acceleration will radiate a thermal bath of particles with a temperature given by the Unruh radiation associated to its value of acceleration, $a$

$$
T_{m m}=T_{\text {unruh }}=\frac{\hbar a}{2 \pi c} .
$$

It is important to note that this is a nontrivial observation, as a moving mirror is a different

physical system than the detectors first studied by Unruh and others. However it is easy to make a thermodynamic argument for it. If I am in a bath of thermal radiation at temperature $T$ and I uncover a mirror, or take one out of my pocket, that mirror will interact with the radiation and come to equilibrium at the same temperature. Any other behavior would contradict the second law of thermodynamics. But since an accelerating observer in the vacuum is known to observe a thermal bath of radiation, the same must apply to this case, otherwise an accelerated observer could violate the laws of thermodynamics. 
The problem of Unruh radiation has also been studied in $A d S$ spacetimes, by Deser and Levin[13, 14], and Jacobson[15], following a seminal work on QFT in AdS spacetimes by Avis, Isham and Storey[16]. They find that there are several natural choices of vacua, corresponding to the fact that $A d S$ spacetime is not globally hyperbolic. The specification of the vacuum state then depends on boundary conditions imposed at timelike infinity. Isham et al, and Deser and Levin study three natural choices of vacua, corresponding to reflected or transmitted boundary conditions at the time like infinity. They find that a particle detector following a timelike worldline in a $d$ spacetime dimensional $A d S$ spacetime will in some cases detect a flux of particles with a thermal or modified thermal spectrum. They further find that there are simple criteria to determine whether the temperature is non-vanishing, and compute its value. These have to do with the embedding of the accelerating trajectory in the flat $d+1$ dimensional spacetime within which the $A d S$ spacetime is embedded. First, the temperature is only non-vanishing when the trajectory in the $d+1$ dimensional spacetime is hyperbolic, so it has horizons in the embedding spacetime, analogous to the Rindler horizon. Then, when the temperature is non-vanishing it is in fact proportional to the $d+1$ dimensional acceleration of the worldline in the $d+1$ dimensional flat embedding spacetime.

$$
T_{\text {unruh }}=\frac{\hbar a_{d+1}}{2 \pi c}
$$

The moving mirror problem has not, to our knowledge, been studied in an $A d S$ spacetime. But by making a similar thermodynamic argument we can arrive at the conclusion that a moving mirror in $A d S$ spacetime will radiate quanta in a thermal or modified thermal spectrum at a temperature given by (11). Suppose a family of observers in ordinary $A d S$ spacetime follows the trajectories that would be followed by freely falling worldlines of an RS brane. By the results of 13, 14, 15 they would see themselves immersed in a bath of radiation with temperature (11). Now suppose they convert some sand they are carrying into mirrors that totally reflect the quanta in question. Then those mirrors must also come to equilibrium at the same temperature (11), otherwise the second law is violated. Thus, an external observer, moving inertially in the $A d S$ spacetime will see the mirrors radiate a flux of quanta at the same temperature.

Of course the energy must come from the observers, who require energy to both continue their motion and construct the mirrors. The energy drained by the radiation created rep- 
resents a kind of purely quantum mechanical radiation reaction. In the case that the brane itself acts as a mirror, the energy must come from energy density stored in the vacuum of the quantum field theory on the brane.

In the previous section we showed that the RS brane is precisely a mirror for a certain set of modes in the bulk five dimensional $A d S$ spacetime, which we called Type II modes. We then have a prediction that the RS brane will radiate into the bulk a gas of thermal Type II gravitons

$$
T_{R S}=\frac{\hbar a_{6}}{2 \pi c}
$$

where $a_{6}$ is the acceleration of a freely falling particle in the RS brane measured in the six dimensional embedding spacetime.

We need now only look at the details to find out what this implies for RS cosmologies.

The RS brane is embedded in $A d S_{5}$ which is an embedding into a flat six dimensional space

$$
d s_{6}^{2}=-d \tilde{y}_{0}^{2}+d \tilde{y}_{1}^{2}+d \tilde{y}_{2}^{2}+d \tilde{y}_{3}^{2}+d \tilde{y}_{4}^{2}-d \tilde{y}_{5}^{2},
$$

with constraint

$$
-\tilde{y}_{0}^{2}+\tilde{y}_{1}^{2}+\tilde{y}_{2}^{2}+\tilde{y}_{3}^{2}+\tilde{y}_{4}^{2}-\tilde{y}_{5}^{2}=-R^{2}
$$

The coordinates can be parameterized by defining

$$
\begin{aligned}
& \tilde{y}_{0}=\frac{1}{2 z}\left(z^{2}+x_{i}^{2}-t^{2}+R^{2}\right) \\
& \tilde{y}_{i}=\frac{R x_{i}}{z} \\
& \tilde{y}_{4}=-\frac{1}{2 z}\left(z^{2}+x_{i}^{2}-t^{2}-R^{2}\right), \\
& \tilde{y}_{5}=\frac{R t}{z}
\end{aligned}
$$

such that the metric can be written as,

$$
d s_{5}^{2}=\frac{R^{2}}{z^{2}}\left(d z^{2}+d x_{i}^{2}-d t^{2}\right),
$$

where index $i$ runs from 1 to 3 . If we define,

$$
z=R e^{y / R}
$$


then it takes the form,

$$
d s_{5}^{2}=d y^{2}+e^{-2 y / R}\left(d x_{i}^{2}-d t^{2}\right) .
$$

To consider the $d S$ embedding, let us introduce a positive constant $\Lambda_{4}$, and take the following coordinate transformation 17, 18, 19]

$$
\begin{aligned}
y & =-\sqrt{\Lambda_{4}} R t^{\prime}-R \log \left(\sinh \frac{\gamma-y^{\prime}}{R}\right), \\
t & =-R \operatorname{coth} \frac{\gamma-y^{\prime}}{R} e^{-\sqrt{\Lambda_{4}} t^{\prime}} \\
x_{i} & =\Lambda_{4} R^{2} x_{i}^{\prime} .
\end{aligned}
$$

The metric becomes

$$
d s_{5}^{2}=d^{2} y^{\prime}+\left(\Lambda_{4} R^{2} \sin ^{2} h \frac{\gamma-y^{\prime}}{R}\right)\left(e^{2 \sqrt{\Lambda_{4}} t^{\prime}} d^{2} x_{i}^{\prime}-d^{2} t^{\prime}\right)
$$

where $\gamma$ is related to the brane tension by,

$$
\tau=\frac{3}{G_{5} R} \operatorname{coth} \frac{\gamma}{R}
$$

It's evident that, at $y^{\prime}=$ const., the metric of the brane describes the standard deSitter spacetime?. We also see that a de Sitter embedding is only possible for $\tau>3 / G_{5} R$. However as can be seen from (28), below, this does not restrict the effective four dimensional cosmological constant.

Now suppose a graviton detector is set at $x_{i}=0$ on the brane. Despite being static with respect to brane world, it is accelerated in both $A d S_{5}$ and the six-dimensional flat space time. Furthermore, the trajectory of the detector can be identified with the trajectory of the brane in $A d S_{5}$ or $M_{6}$. We are particularly interested in the six-dimensional acceleration $a_{6}$. When $y^{\prime}=$ const. and $x_{i}=x_{i}^{\prime}=\tilde{y}_{i}=0$,

$$
-\tilde{y}_{0}^{2}+\tilde{y}_{4}^{2}=R^{2} \cosh ^{2} \frac{\gamma-y^{\prime}}{R}-R^{2} \equiv a_{6}^{-2}
$$

where $a_{6}$ is the constant acceleration in six dimensional flat space time. We see that the trajectory is indeed hyperbolic in the six dimensional spacetime, which means that the temperature will be non-zero. We can also compute the magnitude of the six dimensional acceleration directly by making use of equations (15), (17) and (19) to compute $d^{2} \tilde{y}^{\alpha} / d s^{2}$, where

\footnotetext{
${ }^{5}$ We emphasize that equation $(20)$ is indeed a solution to the action (11). For details, see [17, 19]; for de Sitter embeddings with two rather than one branes, see [18].
} 
$s$ is the proper time of the detector. As $x_{i}^{\prime}=d x_{i}^{\prime}=0$ and $y^{\prime}=$ const., $s=\sqrt{\Lambda_{4}} R \sinh \frac{\gamma-y^{\prime}}{R} t^{\prime}$. It is straightforward to obtain the magnitude of acceleration which is,

$$
\left|a_{6}\right|^{2}=-\left|a_{y_{0}}\right|^{2}+\left|a_{y_{4}}\right|^{2}=\frac{1}{R^{2} \sinh ^{2} \frac{\gamma-y^{\prime}}{R}} .
$$

Thus, by combining this with the results of [13, 14 we conclude that an observer at $y^{\prime}=$ const. and $x_{i}=x_{i}^{\prime}=y_{i}=0$ on a de Sitter brane will detect thermal radiation with the temperature

$$
T=\frac{\hbar a_{6}}{2 \pi c}=\frac{\hbar}{2 \pi c R \sinh \frac{\gamma-y^{\prime}}{R}} .
$$

To find the effective four dimensional cosmological constant $\lambda_{4 e}$ we must renormalize the coordinates so that the metric is in the form of

$$
d s_{\text {brane }}^{2}=e^{2 \sqrt{\lambda_{4 e}\left(y^{\prime}\right)} s} d^{2} w_{i}-d^{2} s .
$$

This requires new coordinates,

$$
\begin{aligned}
d s & =\left(\sqrt{\Lambda_{4}} R \sinh \frac{\gamma-y^{\prime}}{R}\right) d t^{\prime}, \\
d w_{i} & =\left(\sqrt{\Lambda_{4}} R \sinh \frac{\gamma-y^{\prime}}{R}\right) d x_{i}^{\prime} .
\end{aligned}
$$

The effective cosmological constant for a brane positioned at $y^{\prime}$ is then

$$
\lambda_{4 e}=\frac{1}{R^{2} \sin ^{2} h \frac{\gamma-y^{\prime}}{R}} .
$$

We see then that at fixed $y^{\prime}$ the temperature is related to the effective cosmological constant by the usual de Sitter relation,

$$
T=\frac{\hbar \sqrt{\lambda_{4 e}}}{2 \pi c}
$$

By the above thermodynamic argument we then expect that the brane itself will radiate into the modes for which it serves as a mirror, with the same temperature. These are our main results.

We now make several comments on these results.

- We note that (29) is the same as the de Sitter temperature of de Sitter radiation internal to the brane, which is a consequence of the existence of horizons in the de Sitter 
spacetime. However the moving mirror radiation radiates into the five dimensional $A d S$ spacetime. As the brane is the source of that radiation it must result in energy loss from the brane.

- If we consider the Minkowski and $A d S$ embeddings, which have vanishing or negative cosmological constant, respectively, we can easily show that there is no thermal radiation in these cases. To see this, we consider the flat brane which is obtained by fixing $y$ to be constant in (18), and a quick calculation shows that the acceleration in six dimensional spacetime is zero [ implying a vanishing temperature. As far as the $A d S$ brane is concerned, we have the following choice 18,

$$
\begin{aligned}
y & =-\sqrt{-\Lambda_{4}} R x_{3}^{\prime}-R \log \left(\cosh \frac{\gamma-y^{\prime}}{R}\right), \\
x_{3} & =\operatorname{Rtanh} \frac{\gamma-y^{\prime}}{R} e^{-\sqrt{-\Lambda_{4}} x_{3}^{\prime}}, \\
x_{1,2} & =-\Lambda_{4} R^{2} x_{1,2}^{\prime}, \quad t=-\Lambda_{4} R^{2} t^{\prime},
\end{aligned}
$$

such that the metric has the form,

$$
d s_{5}^{2}=d^{2} y^{\prime}+\left(-\Lambda_{4} R^{2} \cosh ^{2} \frac{\gamma-y^{\prime}}{R}\right)\left[e^{-2 \sqrt{-\Lambda_{4}} x_{3}^{\prime}}\left(d x_{1}^{\prime 2}+d x_{2}^{\prime 2}-d t^{\prime 2}\right)+d x_{3}^{\prime 2}\right]
$$

From (15), (17) and (30), we find the six-dimensional acceleration of a detector at a fixed spatial position on the brane is also zero. Thus, the temperature of the brane is given by

$$
T=\frac{\hbar a_{6}}{2 \pi c}=\frac{\hbar \Theta\left(\lambda_{4 e}\right) \sqrt{\lambda_{4 e}}}{2 \pi c} .
$$

- It of course follows from (24) that, if the effective cosmological constant of our universe is positive, all the fields we observe are very weakly coupled to a five dimensional thermal bath of Type II gravitons with a temperature given by (29). Of course, the coupling is extremely weak, as has been discussed by [3] and other authors. But it is not impossible that there may be observable consequences of this prediction.

\footnotetext{
${ }^{6}$ Even so, note the five dimensional acceleration over the $A d S$ background is not zero. The brane is accelerated with respect to the bulk at the rate of $1 / z_{0}$, where $z_{0}(\leq R)$ is the transverse position of the brane.
} 


\section{Conclusion: A Possible Dynamical Mechanism for Radiating away Positive Cosmological Constant}

The results just describe imply that the embedding of a four dimensional de Sitter universe as a brane in the five dimensional Randall-Sundrum cosmology is unstable. The exact channel that the instability goes through depends on the coupling of the quantum fluctuations of the vacuum to the Type II modes and hence on the model used for the vacuum energy. Of course, as noted in [3] the coupling of matter degrees of freedom living on the brane to the Type II modes may be suppressed by various factors. Let us first consider the case where the suppression is insignificant, so that the radiation may be considered to be blackbody. In this case the energy radiated into the bulk per unit time and unit brane volume is given by the usual formula,

$$
\mathcal{R}=c \hbar^{-4} T^{5} \approx \lambda_{4 e}^{5 / 2}
$$

It is not difficult to see that this effect may be significant for the evolution of the early universe. Let us consider a standard inflationary model in which $\tau=\mathcal{V}(\phi)$ where $\phi$ is the inflation field and $\mathcal{V}(\phi)$ its potential. Let $\lambda_{4 e}=1 / L^{2}$ and define the evaporation time scale by

$$
t_{\text {evap }}=\left(\frac{1}{\tau} \frac{d \tau}{d s}\right)^{-1}
$$

Then an adiabatic approximation, in which the solution can be approximated by a series of de Sitter embeddings with slowly decreasing $\lambda_{4 e}$, will be valid so long as

$$
\frac{t_{\text {evap }}}{L}>>1
$$

But it is then not difficult to see that

$$
\frac{t_{\text {evap }}}{L}>3\left(\frac{L}{l_{P l}}\right)^{2}\left(\frac{L}{R}\right)^{2}
$$

so that if $R \approx T e v^{-1}$ to solve the hierarchy problem then an adiabatic approximation is good so long as $L>10^{7} l_{P l}$. At the same time, we see that the evolution of an inflationary solution may be significantly modified at early times.

The rate of decay may be slowed by suppression factors coming from the fact that the coupling of Type II modes to the vacuum energy is weak and gravitational. But the effect 
of these will be to increase the evaporation time, which increases the regime in which an adiabatic approximation is valid.

We note that the effect is present so long as $\lambda_{4 e}>0$, which means that $\tau>3 / G_{5} R$. Thus this effect may radiate away all contributions to the effective cosmological constant, including those that result from matter falling onto the brane from the bulk, such as described in ([20, 21] ). In principle, the radiation continues until $\lambda_{4 e}=0$, at which point $\tau$ decreases to the value necessary to stabilize the embedding of the flat $R S$ brane. However the evaporation time becomes much longer than $L$ as $\lambda_{4 e}$ decreases. More work is then needed to understand whether this effect may be significant for observational astronomy.

In closing we remark on several checks of our reasoning that should be carried out. First, the thermodynamic argument we rely on here should be checked by doing a first principles calculation of radiation from a moving mirror in a five dimensional $A d S$ spacetime. These calculations are in progress 22]. Given these results one may investigate, in the context of particular models of the inflation, whether the effective cosmological constant is in fact sent to zero and how the transition from an inflating universe to a FRW universe takes place. Finally, the question of whether both Type I and Type II modes are genuine linearizations of full solutions to the Einstein equations should be investigated by carrying out the linearizations to higher order.

\section{Acknowledgments}

We are grateful to Daniel Freedman, Kelle Stelle, Emmanual Katz, Janna Levin and Lisa Randall for discussion and correspondence and to Clifford Burgess, Robert Brandenberger, Andrew Chamblin, Gia Dvali, Ted Jacobson, Nemanja Kaloper and Robert Myers for com-

ments on earlier versions of this letter. Yi Ling and Lee Smolin would like to thank Chris Isham and the theoretical physics group at Imperial College for hospitality. This work was supported by the NSF through grant PHY95-14240, a PPARC grant and gifts from the Jesse Phillips Foundation.

\section{References}


[1] N. Arkani-Hamed, S. Dimopoulos and G. Dvali, Phys. Lett. B 429, 263 (1998) hepph/9803315.

[2] I. Antoniadis, N. Arkani-Hamed, S. Dimopoulos and G. Dvali, Phys. Lett. B 436, 257 (1998) [hep-ph/9804398].

[3] L. Randall and R. Sundrum, Phys. Rev. Lett. 83, 4690 (1999) hep-th/9906064.

[4] L. Randall and R. Sundrum, Phys. Rev. Lett. 83, 3370 (1999) hep-ph/9905221.

[5] W. G. Unruh, Phys. Rev. D 14 (1976) 870.

[6] P.C.W.Davies and S.A. Fulling, Proc. R. Soc. Lond. A 348, 393 (1976).

[7] N.D. Birrel and P.C.W.Davies, Quantum fields in Curved Space, Cambridge University Press (1982).

[8] Nathan P. Myhrvold, Print-83-0375 (Princeton), May 1983; Phys. Lett. B 132, 308 (1983); Phys. Rev. D 28, 2439 (1983); L.H. Ford, Phys. Rev. D 31, 710 (1985); N.C. Tsamis and R. Woodard, Nucl. Phys. B 474,235 (1996),hep-ph/9602315; Murat Ozer, M.O. Taha, Mod. Phys. Lett.A 13, 571, (1998) gr-qc/9802023; Robert Brandenberger,hep-th/0004016; N. Tsamis, Phys.Lett. B301 (1993) 351.

[9] A. Chamblin, A. Karch and A. Nayeri, Phys. Lett. B 509, 163 (2001) hep-th/0007060.

[10] C. Deffayet, G. Dvali and G. Gabadadze, astro-ph/0105068.

[11] J. Levin, personal communication.

[12] R. M. Wald, General Relativity, The University of Chicago Press (1984).

[13] S. Deser and O. Levin, Class. Quant. Grav. 14, L163 (1997) gr-qc/9706018.

[14] S. Deser and O. Levin, Class. Quant. Grav. 15, L85 (1998) hep-th/9806223.

[15] T. Jacobson, Class. Quant. Grav. 15, 251 (1998) gr-qc/9709048.

[16] S. J. Avis, C. J. Isham and D. Storey, Phys. Rev. D 18, 3565 (1978). 
[17] N. Kaloper, Phys. Rev. D 60, 123506 (1999) hep-th/9905210.

[18] O. DeWolfe, D. Z. Freedman, S. S. Gubser and A. Karch, Phys. Rev. D 62, 046008 (2000) [hep-th/9909134.

[19] A. Karch and L. Randall, JHEP 0105 (2001) 008, hep-th/0011156].

[20] C. Bruck, M. Dorca, C. Martins, M. Parry, Phys. Lett. B495, 183 (2000).

[21] T. Boehm, R. Durrer, C. Bruck, Dynamical Instabilities of the Randall-Sundrum Model, hep-th/0102144.

[22] Yi Ling, in progress. 